\title{
Brillouin distributed time-domain sensing in optical fibers: state of the art and perspectives
}

\author{
LUC THÉVENAZ (ه)
}

Ecole Polytechnique Fédérale de Lausanne, Institute of Electrical Engineering, Lausanne 1015, Switzerland

(C) Higher Education Press and Springer-Verlag Berlin Heidelberg 2010

\begin{abstract}
Optical fiber sensors based on stimulated Brillouin scattering have now clearly demonstrated their excellent capability for long-range distributed strain and temperature measurements. The fiber is used as sensing element, and a value for temperature and/or strain can be obtained from any point along the fiber. After explaining the principle and presenting the standard implementation, the latest developments in this class of sensors will be introduced, such as the possibility to measure with a spatial resolution of $10 \mathrm{~cm}$ and below while preserving the full accuracy on the determination of temperature and strain.
\end{abstract}

Keywords optical fiber, optical fiber sensors, distributed fiber sensors, stimulated Brillouin scattering, nonlinear fiber optics

\section{Introduction}

Distributed Brillouin sensing was first proposed in the late 1980 s as an alternative technique to the classic optical time-domain reflectometry (OTDR) to measure local attenuation along an optical fiber [1]. It turned out rapidly that it had many more potentialities for sensing, since Brillouin scattering is intrinsically very sensitive to temperature and the deformations experienced by the sensing fiber $[2,3]$. This comes from the property that Brillouin scattering must satisfy a very strict phasematching condition, making the interaction to manifest as a spectrally narrow resonance. For a spontaneous scattering, the scattered light will present a sharp spectrum peaking at a frequency shifted from the pump by the quantity [4]:

$$
v_{\mathrm{B}}=\frac{2 n V_{\mathrm{a}}}{\lambda_{\mathrm{o}}},
$$

Received August 1, 2009; accepted October 30, 2009

E-mail: luc.thevenaz@epfl.ch commonly named Brillouin frequency shift, where $n$ represents the effective refractive index of the propagating mode, $V_{\mathrm{a}}$ is the acoustic velocity in the fiber $(\sim 5800 \mathrm{~m} / \mathrm{s})$, and $\lambda_{\mathrm{o}}$ is the vacuum wavelength of the incident light.

For a stimulated scattering, a weak signal wave will experience a sharp gain if it is spectrally positioned at a frequency shifted by $v_{\mathrm{B}}$ below a strong pump propagating in the opposite direction through the fiber.

As expressed in Eq. (1), the Brillouin frequency shift $v_{\mathrm{B}}$ is very dependent on the acoustic velocity and any change of this velocity will be observed as a spectral shift of the resonance. In a solid, the theory of elasticity gives the following general dependence for the acoustic velocity [4]:

$$
V_{\mathrm{a}}=\sqrt{\frac{K}{\rho}},
$$

where $K$ is the bulk modulus and $\rho$ is the material density.

The density of the material $\rho$ is temperature-dependent as a result of the thermal expansion, so that the peak frequency of the interaction is observed to change with the temperature. This is illustrated in the top part of Fig. 1, showing a measurement of the gain experienced by the signal as a function of the frequency difference between pump and signal, for different temperatures [5].

Any deformation experienced by the fiber will also have an impact on its density $\rho$, and this property is particularly exploited to use the fiber as a distributed strain gauge, by observing the shift of the resonance when the fiber is elongated, as shown in the bottom part of Fig. 1.

Therefore, temperature and strain can be evaluated using Brillouin scattering by determining the frequency difference between pump and signal that gives the maximum interaction. It is remarkable that the relationship between these two quantities and the Brillouin shift $\nu_{\mathrm{B}}$ is very linear, as shown in Fig. 2 [5].

From the point of view of the measuring potentialities, Brillouin-based techniques bring the following advantages over other distributed techniques: 


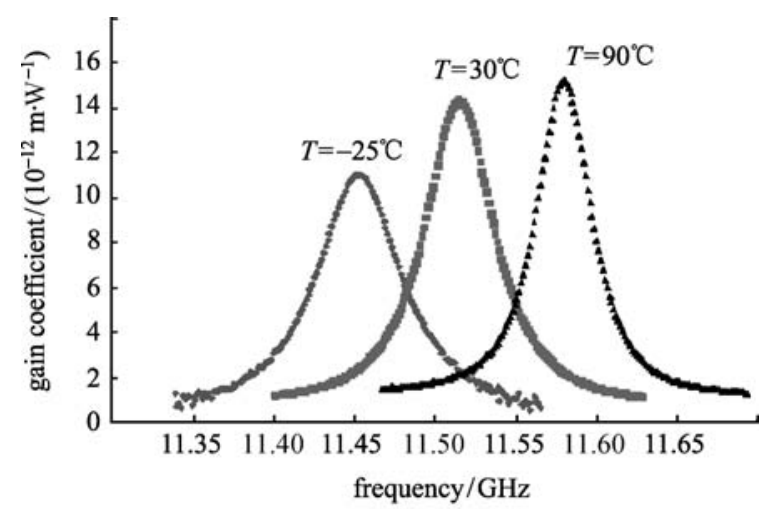

(a)

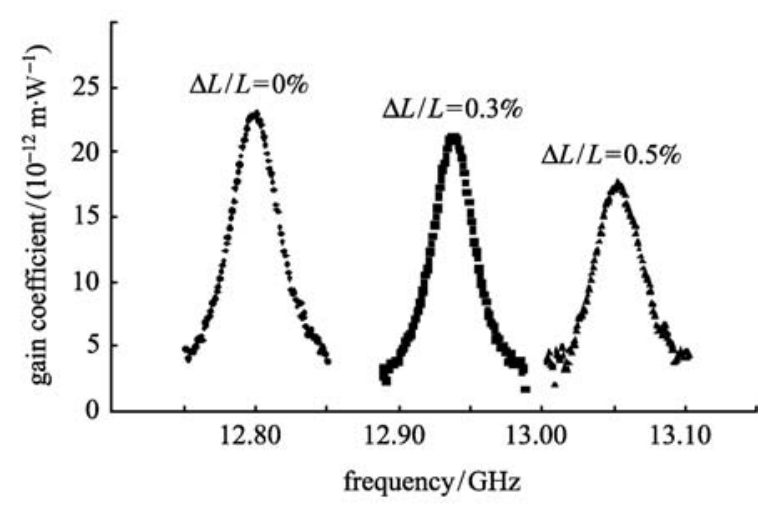

(b)

Fig. 1 Measurement of gain experienced by a signal through stimulated Brillouin scattering as a function of frequency difference between pump and signal. (a) Gain obtained for different temperatures; (b) gain obtained for different longitudinal strains applied to the fiber, showing Brillouin frequency shift dependence on environmental quantities

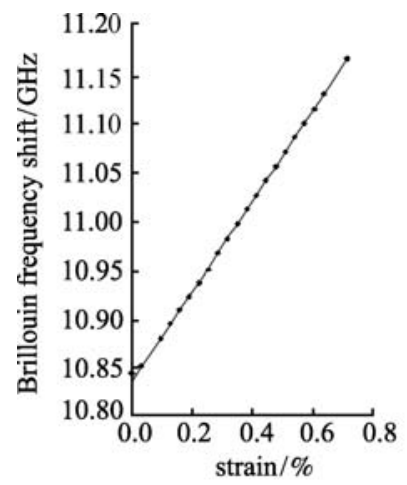

(a)

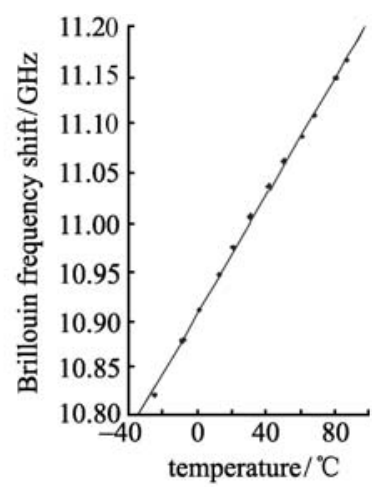

(b)
Fig. 2 Relationship between two quantities and Brillouin shift in a standard single-mode fiber for a $1550 \mathrm{~nm}$ pump wavelength. (a) Longitudinal strain applied to the fiber (strain coefficient: $505.5 \mathrm{MHz} / \%$ ); (b) temperature (temperature coefficient: $\left.0.95 \mathrm{MHz} /{ }^{\circ} \mathrm{C}\right)$

1) The technique makes use of standard low-loss single-mode optical fibers offering several tens of kilometers of distance range and a compatibility with telecommunication components.
2) It is a frequency-based technique as opposed to Raman techniques that are intensity-based. Brillouin techniques are consequently inherently more accurate and more stable on the long-term, since intensity-based techniques suffer from a higher sensitivity to drifts and from a potential biasing by any step loss.

3) Since the information is not a consequence of the background thermal activation, experimental solutions based on stimulated Brillouin scattering can be exploited, leading to a much greater intensity of the scattering mechanism and consequently a more acceptable signal-to-noise ratio.

The different implementations for distributed Brillouin sensing will be reviewed hereafter.

\section{Brillouin optical time-domain reflectometry (BOTDR)}

This technique is very similar to the Rayleigh-based OTDR and the spontaneous Brillouin light backscattered from an intense pulse is recorded as a function of time [6]. To extract the local frequency distribution, the backscattered signal is optically mixed with the continuous wave $(\mathrm{CW})$ light from the laser and the detected beat signal is then electrically mixed with a microwave local oscillator. The recorded signal will then be proportional to the optical beat amplitude at the frequency of the microwave oscillator. By scanning step-by-step the frequency of the microwave oscillator and by recording for each step the mixed signal in the time domain, the frequency distribution of the backscattered signal can then be reconstructed at each position by analyzing for each time step the amplitude as a function of frequency and determining the peak value. A block diagram of a typical BOTDR setup is sketched in Fig. 3.

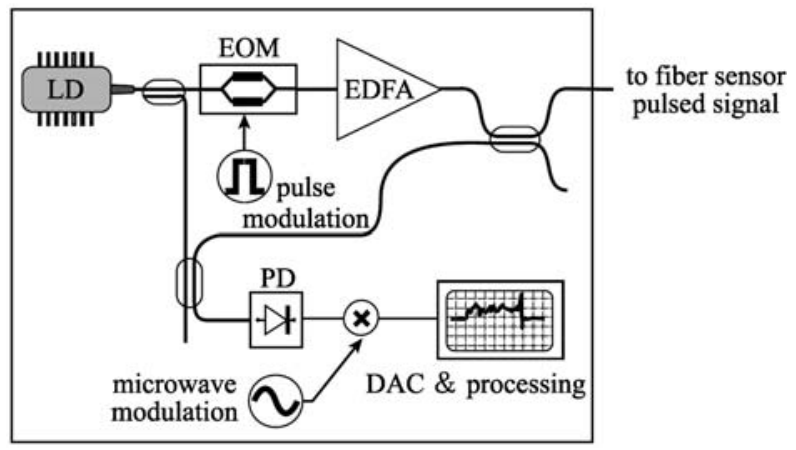

Fig. 3 Block diagram of a typical BOTDR setup

The Brillouin backscattered signal being 100 times smaller than Rayleigh backscattering, the detection scheme must be particularly efficient and sensitive while offering a bandwidth $>10 \mathrm{GHz}$. The backscattered signal, however, benefits from the coherent mixing with the pump and also 
frequently from amplification by the pulse through stimulated Brillouin scattering. This does not have an impact on the measurement accuracy, since the information is not contained in the signal amplitude and such amplification does not bias the frequency content.

The spatial resolution is practically limited to $1 \mathrm{~m}$ for the following reasons: if the pulse spans over a bandwidth larger than the Brillouin gain spectrum, the backscattered signal will show a broader spectral distribution given by the convolution of the natural Brillouin gain distribution with the pulse spectrum. This will smear out the backscattered signal over a wide spectral range and the measurement contrast vanishes. Since the natural Brillouin linewidth is given by the acoustic lifetime of about $10 \mathrm{~ns}$, the activating pulse should not be shorter than this time, corresponding to a spatial resolution of $1 \mathrm{~m}$.

The BOTDR is very attractive in some field environment since it requires the access to a single fiber end. The distance range is $10 \mathrm{~km}$ for a $1 \mathrm{~m}$ spatial resolution and is increased to $30 \mathrm{~km}$ for a $2 \mathrm{~m}$ spatial resolution. These figures are obtained by averaging the signal $2^{14}=16384$ times and can be improved by regenerating the signal through Raman or erbium-doped fiber amplification in the sensing fiber, at the expense of a more complicated setup [7].

\section{Brillouin optical time-domain analysis (BOTDA)}

This technique takes advantage of the stimulated version of Brillouin scattering, a nonlinear interaction, and is based on a pump-probe technique: an intense pump pulse will interact locally during its propagation with a weak $\mathrm{CW}$ probe and the gain experienced by the probe on each location can be analyzed by recording the probe amplitude in the time domain. The frequency difference between pump and probe is scanned step-by-step and the local amplification can be retrieved for a given pump-probe frequency difference. The local gain spectrum can then be reconstructed by analyzing the gain at a given location as a function of frequency, as shown in Fig. 4.

The main difficulty is to generate a pump and a probe with a fixed and stable frequency difference of about $10 \mathrm{GHz}$, with stability typically better than $1 \mathrm{MHz}$. The first demonstrations used two distinct lasers that were frequency locked using a servo loop $[2,3,8]$. This is an expensive solution that is subject to instabilities in an adverse environment. Nowadays, most solutions are based on a single laser source that is modulated to make the pump pulses [9]. The light from the same laser is used to synthesize the probe wave by using an electro-optic modulator driven by a microwave signal at the target frequency [5]. The modulator is biased to operate in a suppressed carrier scheme, so that the lower modulation sideband can be used as probe signal (after suppressing the

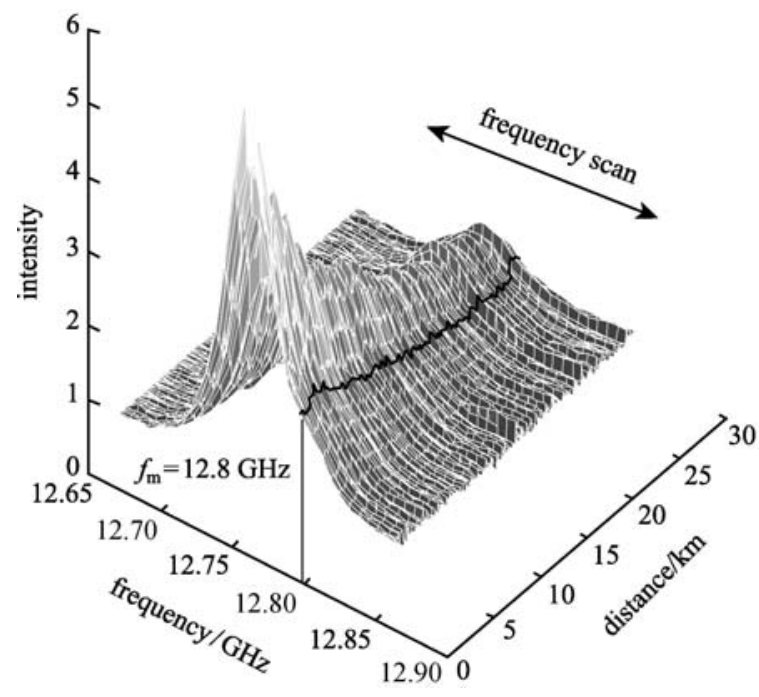

Fig. 4 Principle of data acquisition in a BOTDA system (local amplification is retrieved in time domain and converted into distance units; typical time trace for a pump-probe frequency difference set at $12.800 \mathrm{GHz}$ is highlighted; local gain spectrum at each position can then be reconstructed after a full frequency scan)

upper sideband by optical filtering) [10]. This scheme offers ideal stability since any frequency drift of the laser has no impact on the frequency difference between pump and probe. A scheme of a typical BOTDA system is shown in Fig. 5.

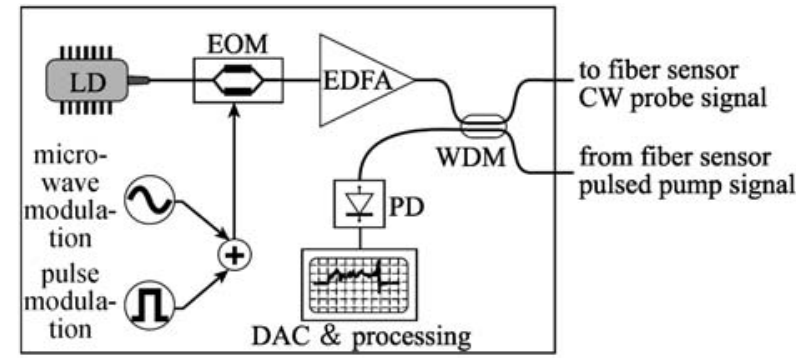

Fig. 5 Block diagram of a typical BOTDA setup

BOTDA setup requires the access to both fiber ends since pump pulse and $\mathrm{CW}$ probe must counterpropagate in the sensing fiber. This can be judged as a limitation in some situations. In addition, the electrostriction that stimulates the acoustic wave is driven by the interference between pump and signal, so that their states of polarization must be preferably aligned to create the maximum gain. Orthogonal polarizations will result in a totally vanishing gain, and since the polarization normally varies randomly along an optical fiber [11], a non-zero gain can only be secured using polarization scrambling or a polarization-diversity scheme. This polarization dependence can also be favorably used to efficiently and rapidly measure the local birefringence properties along an optical fiber [12].

The BOTDA is subject to the same limitation as 
BOTDR for the spatial resolution, i.e., $1 \mathrm{~m}$, limited by the gain spectrum spreading due to the pump spectral broadening for short pulses. This $1 \mathrm{~m}$ spatial resolution can be secured up to a distance of $30 \mathrm{~km}$ and requires an averaging of less than 1000 to get performances identical to the BOTDR. Figure 6 illustrates the capacity of such a system, showing the local elongation of a short segment of fiber, the raw signal amplification in the time domain for each frequency step, visualized in a three-dimensional (3D) graph, and finally, after processing, the value of the strain as a function of the position.

The BOTDA configuration is still under development and is showing constant progress: some solutions are proposed to extend the range and improve the spatial resolution. However, stimulated Brillouin scattering offers the possibility of very innovative schemes and a couple of them will be presented hereafter.

The record spatial resolution is currently obtained by a correlation technique proposed in 2000 by Hotate et al. from the University of Tokyo $[13,14]$. In this elegant alternative technique, the correlation between two continuous lightwaves is controlled. This way, Brillouin scattering can be generated locally along the fiber and the use of a pulsed lightwave is no longer required. Frequency modulation (FM) is the technique that was implemented to create local correlations, and centimetric spatial resolutions can be routinely obtained, however, over a limited distance.

Nearly equivalent spatial resolutions can nevertheless be obtained today using pure time-domain techniques over kilometric ranges that will be presented in the following sections.

\section{Brillouin echo distributed sensing (BEDS)}

The commonly accepted opinion that the spatial resolution of a pulse-based Brillouin sensor is limited to $1 \mathrm{~m}$ was seriously questioned when Bao et al. observed an unexpected narrowing of the Brillouin gain spectrum down to its natural linewidth when pulses turned shorter than the acoustic lifetime (10 ns) [15]. This breakthrough opened the incredible perspective of performing high spatial resolution measurement while maintaining a sharp Brillouin resonance.

It is now unanimously accepted by all specialists that this special behavior results from a pre-excitation of the acoustic wave through the presence of a continuous background pump [16]. This was originally observed by using a modulator with a poor off-state extinction ratio to form the pump pulses.

Basically, the observation of this effect depends on the pre-existence of an important acoustic wave in the fiber medium vibrating at the exact Brillouin frequency resonance. This acoustic wave is conveniently generated through stimulated Brillouin scattering using a continuous wave at the probe frequency and another continuous wave (or a long pulse) at the pump frequency. Among the three waves involved in the interaction, the two optical waves can experience very fast changes in their amplitude and phase, while the acoustic wave presents a highly inertial behavior and requires a typical time equal to its $10 \mathrm{~ns}$ lifetime to adapt to a new situation. For instance, if the pump is suddenly turned off, the acoustic wave will gradually decay and will still exist during the typical duration of its lifetime despite the absence of stimulated interaction.

This inertial property is used to change very briefly amplitude or phase of the pump, during a time $T$ so short that the acoustic wave does not experience a notable change ( $T \ll$ acoustic lifetime). This way, the pump will be reflected by the acoustic wave but under transient conditions that do not correspond to those required by amplitude and phase matching. This will cause a fast change of the steady-state amplification of the probe that can be observed by a fast detection scheme. The preexistence of the acoustic wave is essential, and since it is stimulated by continuous optical waves, a response will be

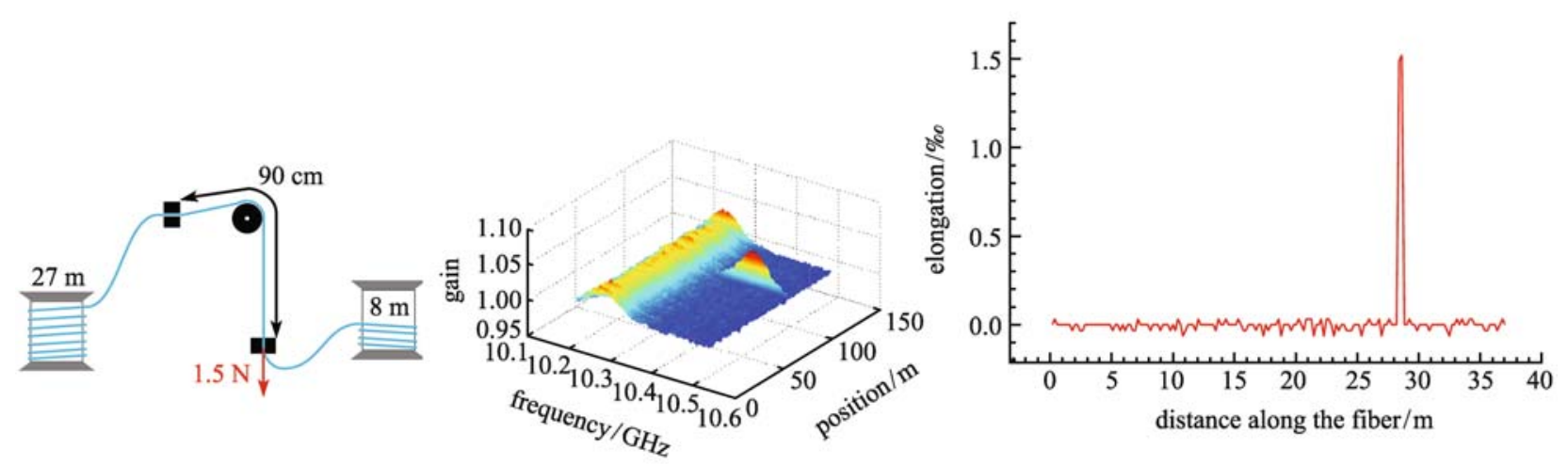

(a)

(b)

(c)

Fig. 6 BOTDA measurement of a short strained segment. (a) Experimental layout; (b) 3D graph of raw signal amplification in time domain, while frequency is scanned; (c) strain distribution calculated from raw signal amplification 
observed only over the natural local Brillouin gain spectrum along the fiber.

To highlight the similarity with spin echoes, this new concept is named Brillouin echo because it turns out to be formally very similar to the description of the interaction of nuclear spins with magnetic fields in nuclear magnetic resonance.

The Brillouin echo can be observed essentially with three different pump pulse codings, detailed here below:

1) Bright pulse (as shown in Fig. 7): The effect was first observed using this configuration [15]. The finite extinction ratio of the electro-optic modulator lets a continuous flow of pump light activate the acoustic wave, giving constant background amplification on the probe (dark topping). The additional pump light in the pulse is reflected and its effect is observed as an additional amplification. This configuration is the less efficient if the peak power of the pump is fixed.

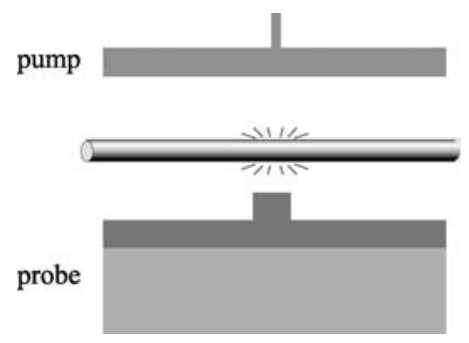

Fig. 7 Configuration of bright pulse

2) Dark pulse (as shown in Fig. 8): The pump is turned off during a short time, so that no light is reflected and the constant background amplification is stopped [17]. The acoustic wave keeps vibrating during the dark pulse and the background amplification is restored when the pump light is on again. The effect of the dark pulse is observed as a "negative" gain (actually, it is an absence of gain).

3) Phase pulse (as shown in Fig. 9): A $\pi$-phase shift is applied on the pump for a short time, so that the

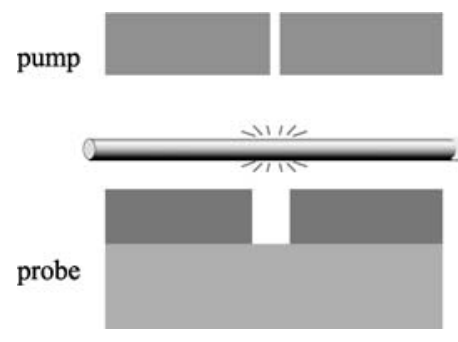

Fig. 8 Configuration of dark pulse

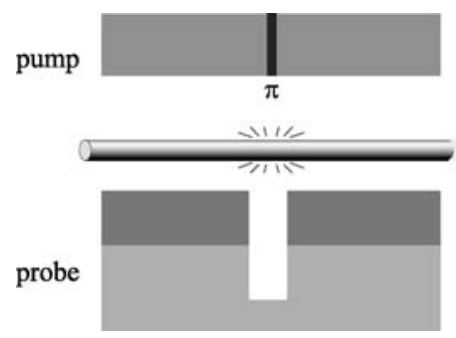

Fig. 9 Configuration of phase pulse

reflected light interferes destructively with the signal, equivalent to a Brillouin loss process [18,19]. The response is identical to the dark pulse but is twice larger for an identical pump power and is thus the most efficient [20].

The setup implementation is very similar to a BOTDA configuration as shown in Fig. 10. For the first two situations, the pump pulses are also shaped by an intensity electro-optic modulator that is simply electrically biased differently. In the case of the $\pi$-phase pulse, a phase modulator substitutes for the intensity modulator.

The most important problem faced by such a technique is the fact that the acoustic wave is partially decaying during the pulse duration. This creates a second attenuated echo when the pump is restored to its original state after the pulse, but this echo extends over a duration given by the acoustic lifetime and thus creates a ghost response over the

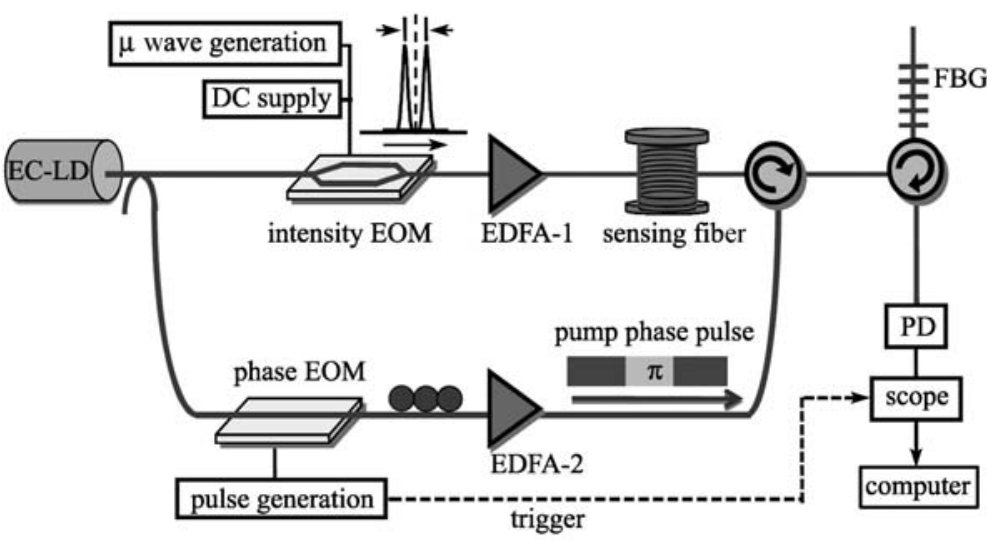

Fig. 10 Block diagram of a BEDS setup based on a $\pi$-phase modulation of the pump 
subsequent measured points [20]. Two solutions have been identified to attenuate or even to totally suppress the impact of the second echo:

1) By deconvolving numerically the time traces: The impulse response of any BEDS sensor has been analytically determined, and in particular, the contribution of the second echo to this impulse response can be unambiguously extracted [20]. This contribution can then be removed from the time traces by a simple step-by-step single-pass algorithm.

2) By turning off the pump immediately after the pump phase pulse: No more light from the pump can be reflected after the pulse end and no trailing light is present. This solution was successfully tested [19] for a phase pulse in a differential measurement configuration by subtracting the traces obtained with the phase pulse turned on and off, successively, and was recently adapted for a bright pulse [21].

Figure 11 shows the distribution of Brillouin amplification over the last $40 \mathrm{~m}$ of a $4.57 \mathrm{~km}$ fiber, obtained using a $500 \mathrm{ps} \pi$-phase pulse in the differential measurement technique described above. A $5 \mathrm{~cm}$ segment showing a distinct Brillouin shift can be clearly identified and measured even after several kilometers of fiber, as shown in Fig. 12. The accuracy on the Brillouin shift determination remains comparable to a standard BOTDA technique, since no significant broadening of the measured Brillouin spectrum is observed.

\section{Brillouin dynamic grating distributed sensing (BDG-DS)}

In recent studies [22-24], the concept of Brillouin dynamic grating (BDG) has been newly implemented in polarizationmaintaining fibers (PMFs), where acoustic waves

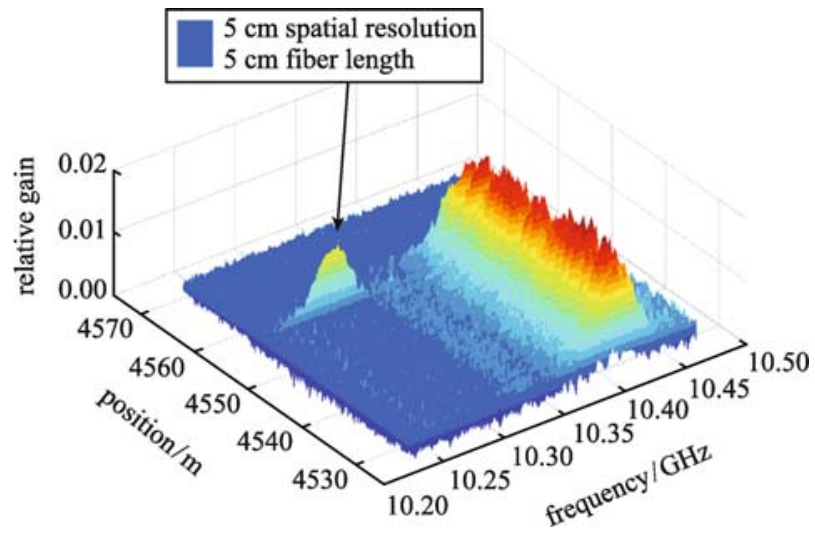

Fig. 11 3D view of signal amplification obtained using BEDS technique in a differential configuration with $500 \mathrm{ps} \pi$-phase shifts on the pump (here are shown the 40 last meters of a $4.57 \mathrm{~km}$ fiber, where a $5 \mathrm{~cm}$ segment showing a distinct Brillouin shift is inserted)

generated during the process of stimulated Brillouin scattering (SBS) by optical waves (pump waves) in one polarization are used to reflect an orthogonally polarized wave (probe wave) at a different optical frequency from the pump. As shown in the conceptual scheme in Fig. 13, the frequency separation $\Delta f$ between the pump and the probe waves in the BDG operation is determined by the birefringence of the PMF and lies in the several tens of $\mathrm{GHz}$ in usual cases. As a potential application, a novel type of birefringence-based distributed sensor has been proposed using the BDG with an order-of-magnitude higher sensitivity in the temperature measurement compared to ordinary Brillouin sensors [23-25].

BDG can also be applied to enhance the spatial resolution of an ordinary Brillouin optical time-domain analysis (BOTDA) system by replacing the Brillouin probe with the reflection from the BDG. It is possible this way to

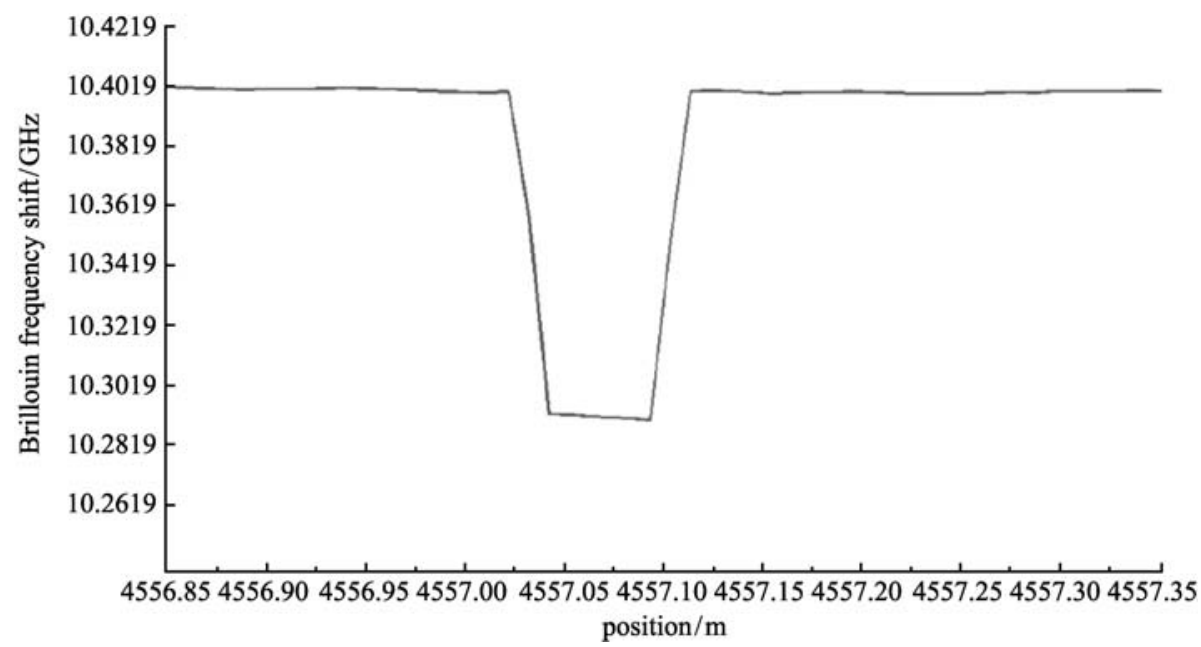

Fig. 12 Distribution of Brillouin frequency shift at position of this segment obtained after processing, showing that the segment is clearly resolved (horizontal axis limited to a $1.5 \mathrm{~m}$ range) 


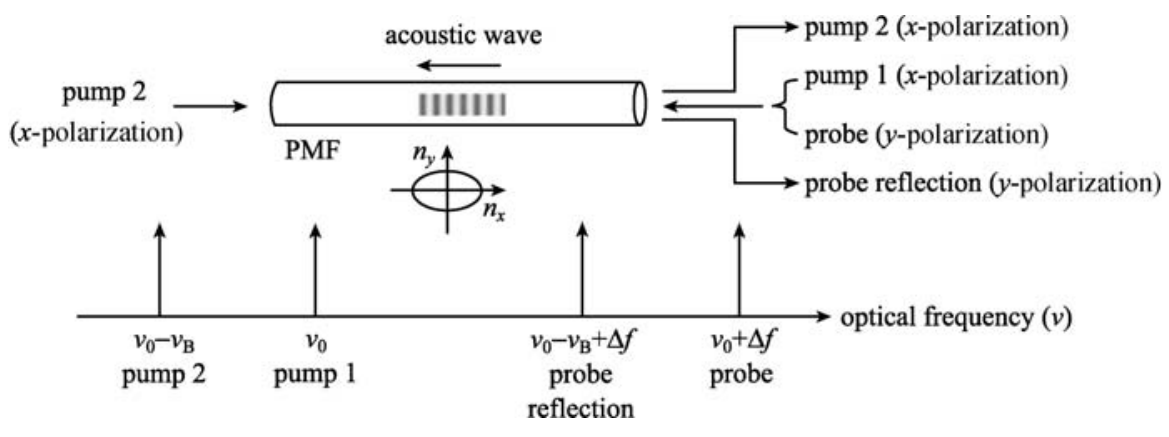

Fig. 13 Description of concept for generation and reading of Brillouin dynamic grating

acquire a narrowband Brillouin gain spectrum (BGS) with a broadband pulse using the BDG, and measurements with a $1 \mathrm{~cm}$ spatial resolution have been demonstrated experimentally, which is the best result ever reported using a time-domain Brillouin sensor [26].

Figure 14 shows the principle of the BDG distributed sensing where the pump waves (pump 1 and pump 2) in the $x$-polarization and a probe pulse in the $y$-polarization are applied for the generation of the BDG (i.e., acoustic wave) and its readout, respectively. In this scheme, a relatively long pulse $(30 \mathrm{~ns})$ for pump 1 is used to maintain a narrowband Brillouin gain but meanwhile adopt an ultrashort probe pulse $(\sim 110 \mathrm{ps})$ to realize a high-resolution acquisition of the local Brillouin gain spectrum.

When the broadband probe pulse is reflected by the local

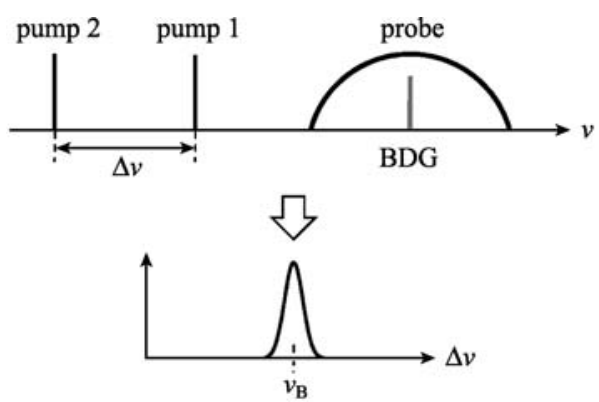

Fig. 14 Acquisition of a local Brillouin gain spectrum with a short pulse in a BDG-based distributed sensing system through BDG strength variation
BDG, the reflectance, which is determined by the spectral overlap between the BDG and the probe pulse, is proportional to the strength of the BDG. It means that the time trace of the probe reflection shows the local strength of the BDG, which depends on the Brillouin gain in the process of SBS between the two pump waves. Thus, the shape of the BGS can be retrieved from the probe reflection by sweeping the frequency offset between pump 1 and pump 2. Since the spatial resolution of this measurement is determined by the duration of the probe pulse, while the bandwidth of the Brillouin gain is related to the duration of pump 1, a high spatial resolution can be achieved while keeping a narrow effective Brillouin gain spectrum. It must be pointed out that the probe frequency is not scanned, since its spectrum covers a much broader spectrum than any reasonable change of the BDG frequency due to environment, as depicted in Fig. 14.

A typical implementation of a BDG-based system is shown in Fig. 15. It must be pointed out that pump 1 must be preferably pulsed to avoid any depletion and an excessive gain for pump 2, with a duration far exceeding the acoustic lifetime ( $>10 \mathrm{~ns})$.

A measurement of a $1.5 \mathrm{~cm}$ hotspot is presented in Fig. 16, obtained using $110 \mathrm{ps}$ probe pulse. The smooth transitions are not due to a limitation in the resolving power but to the natural thermal transfer along the optical fiber that can be observed in this situation.

This shows the huge progresses achieved these past few years in Brillouin time-distributed sensing, demonstrating

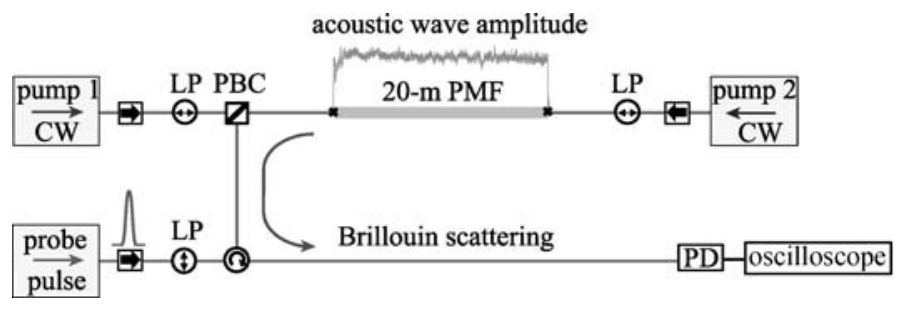

Fig. 15 Block diagram of a typical implementation of BDG-DS setup (pump 1 and pump 2 can be generated from the same laser using a similar technique as in a BOTDA system based on modulation sideband; LP: linear polarizer; PBC: polarization beam combiner; PD: photodiode) 


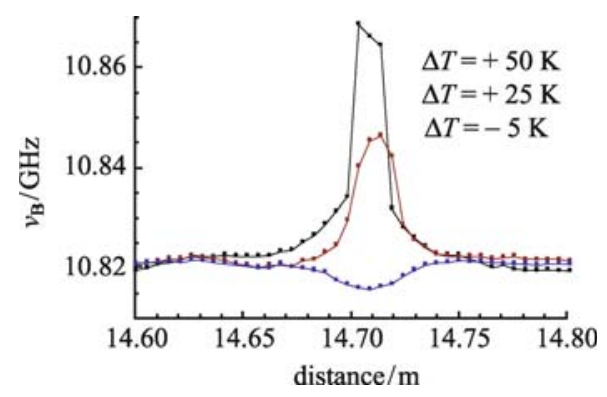

Fig. 16 Measurement of a $1.5 \mathrm{~cm}$ spot placed at different temperatures with a BDG-DS system using $110 \mathrm{ps}$ probe pulses

the possibility to resolve events with a spatial resolution in the centimeter range. This is fully comparable to point sensors such as fiber Bragg gratings, however, showing the great flexibility of a fully continuous distributed sensing, equivalent to many thousands of distinct point sensors and requiring no special fiber preparation.

Acknowledgements The author is particularly grateful to his past and present collaborators, Marc Niklès, Alexandre Fellay, Massimo Facchini, Dario Alasia, Stella Foaleng Mafang, Sanghoon Chin, and Nikolay Primerov, for their essential contributions to illustrate this report. A special credit is addressed to two visiting scientists, Moshe Tur from Tel-Aviv University and Kwang-Yong Song from Chung-Ang University in Seoul, for their key scientific support. This work was supported by the company Omnisens and by the COST Action 299 "FiDES".

\section{References}

1. Horiguchi T, Tateda M. Optical-fiber-attenuation investigation using stimulated Brillouin scattering between a pulse and a continuous wave. Optics Letters, 1989, 14(8): 408-410

2. Horiguchi T, Kurashima T, Tateda M. A technique to measure distributed strain in optical fibers. IEEE Photonics Technology Letters, 1990, 2(5): 352-354

3. Kurashima T, Horiguchi T, Tateda M. Distributed-temperature sensing using stimulated Brillouin scattering in optical silica fibers. Optics Letters, 1990, 15(18): 1038-1040

4. Horiguchi T, Kurashima T, Tateda M. Tensile strain dependence of Brillouin frequency shift in silica optical fibers. IEEE Photonics Technology Letters, 1989, 1(5): 107-108

5. Nikles M, Thevenaz L, Robert $P$ A. Brillouin gain spectrum characterization in single-mode optical fibers. Journal of Lightwave Technology, 1997, 15(10): 1842-1851

6. Horiguchi T, Shimizu K, Kurashima T, Tateda M, Koyamada Y. Development of a distributed sensing technique using Brillouin scattering. Journal of Lightwave Technology, 1995, 13(7): 12961302

7. Alahbabi M N, Cho Y T, Newson T P. 150-km-range distributed temperature sensor based on coherent detection of spontaneous Brillouin backscatter and in-line Raman amplification. Journal of the Optical Society of America B, 2005, 22(6): 1321-1324

8. Bao X, Webb D J, Jackson D A. 32-km distributed temperature sensor based on Brillouin loss in an optical fiber. Optics Letters, 1993, 18(18): 1561-1563

9. Nikles M, Thevenaz L, Robert P A. Simple distributed fiber sensor based on Brillouin gain spectrum analysis. Optics Letters, 1996, 21 (10): 758-760

10. Thevenaz L, Nikles M, Fellay A, Facchini M, Robert P A. Applications of distributed Brillouin fiber sensing. In: Proceedings of International Conference on Applied Optical Metrology. Balatonfured: SPIE, 1998, 3407: 374-381

11. Van Deventer M O, Boot A J. Polarization properties of stimulated Brillouin scattering in single-mode fibers. Journal of Lightwave Technology, 1994, 12(4): 585-590

12. Thevenaz L, Foaleng-Mafang S, Nikles M. Fast measurement of local PMD with high spatial resolution using stimulated Brillouin scattering. In: Proceedings of the 33rd European Conference on Optical Communication. 2007, 10.1.2

13. Hotate K, Hasegawa T. Measurement of Brillouin gain spectrum distribution along an optical fiber using a correlation-based technique-proposal, experiment and simulation. IEICE Transactions on Electronics, 2000, E83-C(3): 405-412

14. Hotate K, Tanaka M. Distributed fiber Brillouin strain sensing with $1-\mathrm{cm}$ spatial resolution by correlation-based continuous-wave technique. IEEE Photonics Technology Letters, 2002, 14(2): 179181

15. Bao X, Brown A, DeMerchant M, Smith J. Characterization of the Brillouin-loss spectrum of single-mode fibers by use of very short ( $<10-n s$ ) pulses. Optics Letters, 1999, 24(8): 510-512

16. Lecoeuche V, Webb D J, Pannell C N, Jackson D A. Transient response in high-resolution Brillouin-based distributed sensing using probe pulses shorter than the acoustic relaxation time. Optics Letters, 2000, 25(3): 156-158

17. Brown A W, Colpitts B G, Brown K. Dark-pulse Brillouin optical time-domain sensor with $20-\mathrm{mm}$ spatial resolution. Journal of Lightwave Technology, 2007, 25(1): 381-386

18. Foaleng-Mafang S, Beugnot J C, Thevenaz L. Optimized configuration for high resolution distributed sensing using Brillouin echoes. In: Proceedings of the 20th International Conference on Optical Fibre Sensors, Edinburgh: SPIE, 2009, 7503: 75032C

19. Thevenaz L, Foaleng-Mafang S. Distributed fiber sensing using Brillouin echoes. In: Proceedings of the 19th International Conference on Optical Fibre Sensors. Perth: SPIE, 2008, 7004: $70043 \mathrm{~N}$

20. Thevenaz L, Beugnot J C. General analytical model for distributed Brillouin sensors with sub-meter spatial resolution. In: Proceedings of the 20th International Conference on Optical Fibre Sensors. Edinburgh: SPIE, 2009, 7503: 75036A

21. Li W, Bao X, Li Y, Chen L. Differential pulse-width pair BOTDA for high spatial resolution sensing. Optics Express, 2008, 16(26): 21616-21625

22. Song K Y, Zou W, He Z, Hotate K. All-optical dynamic grating generation based on Brillouin scattering in polarization-maintaining fiber. Optics Letters, 2008, 33(9): 926-928

23. Dong Y, Bao X, Chen L. Distributed temperature sensing based on birefringence effect on transient Brillouin grating in a polarizationmaintaining photonic crystal fiber. Optics Letters, 2009, 34(17): $2590-2592$ 
24. Song K Y, Zou W, He Z, Hotate K. Optical time-domain measurement of Brillouin dynamic grating spectrum in a polarization-maintaining fiber. Optics Letters, 2009, 34(9): 1381-1383

25. Zou W, He Z, Song K Y, Hotate K. Correlation-based distributed measurement of a dynamic grating spectrum generated in stimulated Brillouin scattering in a polarization-maintaining optical fiber.
Optics Letters, 2009, 34(7): 1126-1128

26. Song K Y, Chin S, Primerov N, Thevenaz L. Time-domain distributed sensor with $1 \mathrm{~cm}$ spatial resolution based on Brillouin dynamic gratings. In: Proceedings of the 20th International Conference on Optical Fibre Sensors. Edinburgh: SPIE, 2009, 7503: $75037 \mathrm{~V}$ 